\title{
3D structures of steady flow of ideal compressible fluid in MHD: a new model of primordial solar nebula
}

\author{
Vladimir V. Salmin \\ Siberian Federal University, Svobodny ave., 79, Krasnoyarsk, 660041, Russia \\ email: vsalmin@gmail.com
}

\begin{abstract}
Stereographic projection of Hopf field on the 3-sphere into Euclidean 3-space is used as a model of 3D steady flow of ideal compressible fluid in MHD. In such case, flow lines are Villarceau circles lying on tori corresponding to the levels of Bernoulli function. Existence of an optimal torus with minimal relative surface free energy is shown. Optimal tori are considered as precursors of planetary orbits.
\end{abstract}

Keywords. MHD - solar system: formation

\section{Introduction}

The equations of ideal incompressible magnetohydrodynamics (Landau \& Lifshitz 1959) for stationary flow are clearly satisfied when the fluid moves along the magnetic field lines with velocity (see Chandrasekhar 1961). If density is constant it is easy to show that the equation of ideal magnetohydrodynamics can be rewritten in the stationary Euler equation form. In such case, $\vec{v}$ and $\nabla \times \vec{v}$ are tangential to surfaces of Bernoulli function level. Structural theorem of Arnold \& Khesin (1998) determines that if $\alpha$-Bernoulli function for steady flow $\vec{v}$ on orientable 3-manifold $M$ without boundary, and $\Gamma \subset M$ is preimage of critical value $\nabla \alpha=0$, every connected component of the set $M \backslash \Gamma$ is fibered into two-dimensional tori invariant under the flow of $\vec{v}$, the motion on each torus is quasi-periodic.

Solution of the equations of ideal magnetohydrodynamics describes a localized topological soliton with use of Hopf mapping shown by Kamchatnov (1982). Hopf field on $S^{3}$ has minimal energy among all the fields diffeomorphic to it (Arnold \& Khesin 1998). Stability of Hopf field on $S^{3}$ has been proved by (Gil-Merdano \& Llinares-Fuster 2001, Yampolsky 2003). Hopf fibration of $S^{3}$ with stereographic projection induces the toroidal coordinates on $E^{3}$ (Gibbons 2006).

\section{Results}

On a torus $\nu=\operatorname{arcsinh}(k) / k$ stress tensor for steady flow of ideal compressible fluid in toroidal coordinates is determined by metrical tensor only

$$
\left.p^{i j}\right|_{T}=-p \delta_{i}^{j}\left(\sqrt{k^{2}+1}-\cos (k \alpha)\right)^{2} / R^{2} k^{2} .
$$

It is easy to show that the pressure on the torus surface $P \propto r^{-2}$ where $r$-distance from the torus axis to the point on the torus. Let's define cartesian torus form factor $g=a / c$ where $c$ is main torus radius, and $a$-tube radius. Notice that $g=\sin (\theta)$ where $\theta$ inclination of Villarceau circles to the main plane of torus symmetry, we call this angle as "stream inclination". The form factor $g=(\sqrt{2})^{-1} \approx 0.7071 \ldots$ when the ratio of the solid 
torus volume to the force of pressure on it's boundary has maximum. We will call the torus meeting form factor as "optimal torus". Physically, it corresponds to minimal relative surface free energy. Beat of oscillations with wave numbers corresponding to structural radii of optimal torus leads to spatial scaling with scaling factor $K=1+g$. Spatial intersection of homothetic tori within one torus forms cluster with the size depending on a scaling factor.

It is easy to show that scaling factor of solar system corresponds to optimal torus precisely. Scaling factors of satellite systems have deviations depending on their axial tilt and local ratio of semi-major axes of neighbor planets. Large axial-tilt of Uranus is well explained with the suggested model. We found that the cluster size of solar, Jupiter's, Uranus' systems $C=4$, but for Saturn's, Neptune's, Pluto's and Mars' systems $C=3$.

At least two stages of toroidal flow development have been determined in nebula. At first stage, nebula was uniform, and tori separation brought about linear dependence of logarithm of relative planets' volumes on logarithm of relative semi-major axes. Coefficient of proportionality called dimensionality of accumulation ranged from $Z=3.067$ for solar system to $Z=4.413$ for Neptune's satellites. At second stage, main stream of toroidal flow located on tori has resulted in formation of paired planets with neighbor orbits and equal volumes. Number of pairs depended on a value of cluster size. If cluster size was $C=4$, two pairs could be observed, while one pair could be seen when $C=3$.

We found that Triton's parameters fit well the dependence of logarithm of relative volumes on logarithm of relative semi-major axes of regular Neptune's satellites. This means that formation of Triton and regular Neptune's satellites was simultaneous. We have shown that orbit inclination of Triton corresponded to its formation in opposite phase to regular satellites being similar to Pluto's system.

All regular satellites of giant planets with increasing volumes at increasing orbital semi-major axes have distribution of logarithms of relative volumes on the logarithms of relative semi-major axes approximated with linear dependence and high degree of correlation. In suggestion on absence of dissipation of particles involved in toroidal flow around planets, high correlation confirms that density of particles in all satellite systems was similar. Moon has closest parameters to concerned distribution, thereby suggesting that its formation has been processed by a common mechanism.

\section{References}

Landau, L. D. \& Lifhitz, E. M. 1959, Electrodynamics of continious media, Pergamon Press, Oxford

Chandrasekhar, S. 1961, Hydrodynamic and Hydromagnetic Stability, Oxford, Clarendon Press Kamchatnov, A. M. 1982, Sov. JETP 82, No1, 117

Arnold, V. I. \& Khesin, B. A. 1998, Topological Methods in Hydrodynamics, Springer-Verlag, New York

Gil-Merdano, O. \& Llinares-Fuster, E. 2001, Math. Ann. 320, 531

Yampolsky, A. 2003, Acta Math. Hungar. 101, 73

Gibbons, G. W. 2006, Applications of Differential Geometry to Physics Part III, Cambridge University, U.K. 

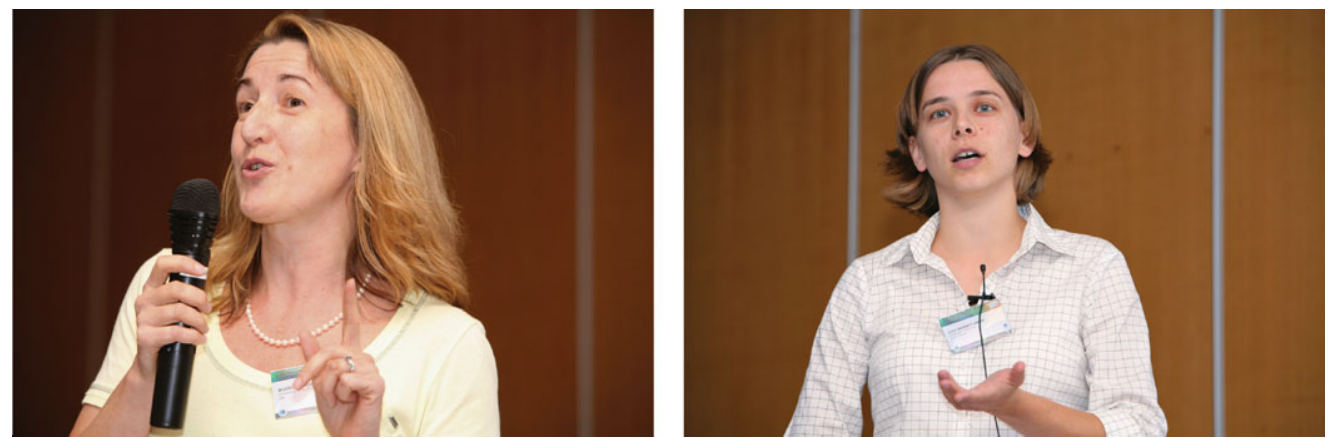

First of the three poster-session chairpersons: Moira Jardine (left). First presenter: Lisa Harvey-Smith (right)
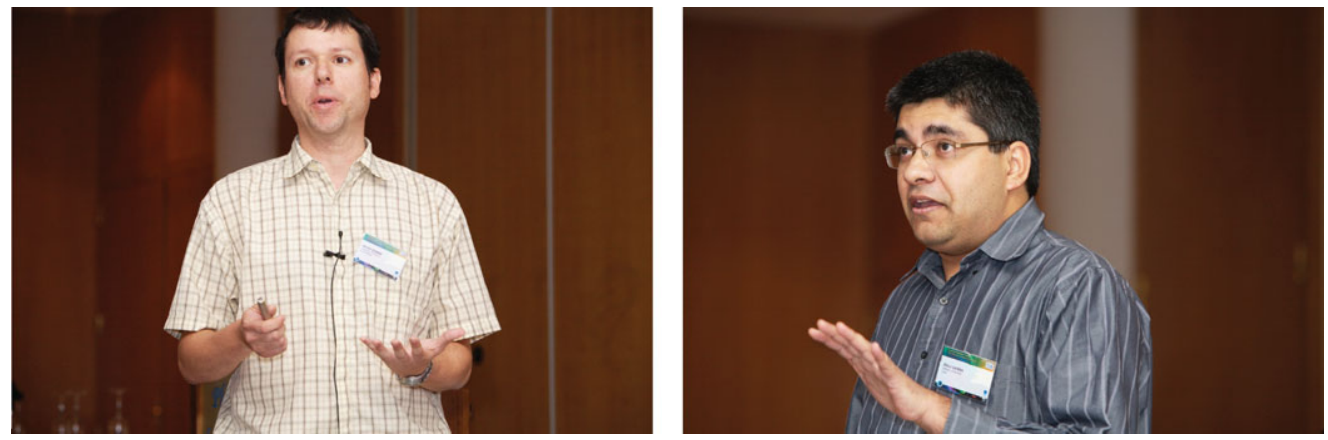

Peter Dobbie (left), and Anuj Sarma (right)
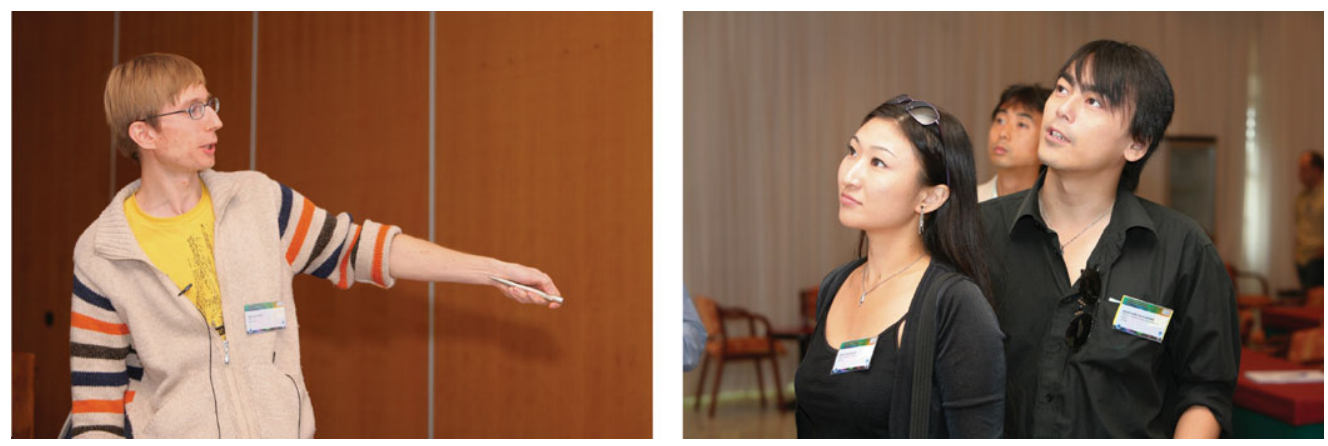

Rainer Arlt (left), and Shio Kawagoe and Nobuhiko Kusakabe (right)
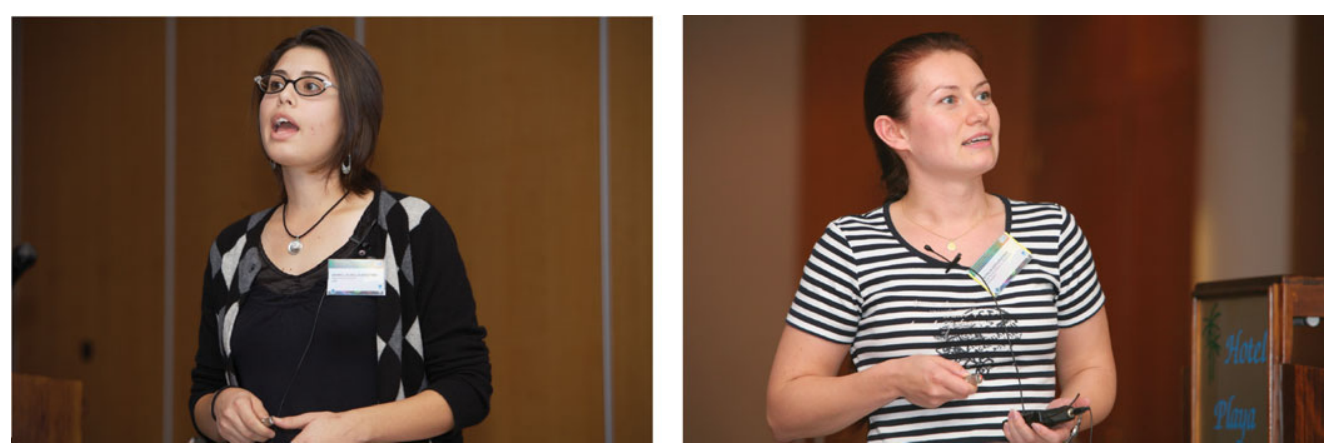

Daniella Della-Giustina (left), and Natalie Dzyurkevich (right) 

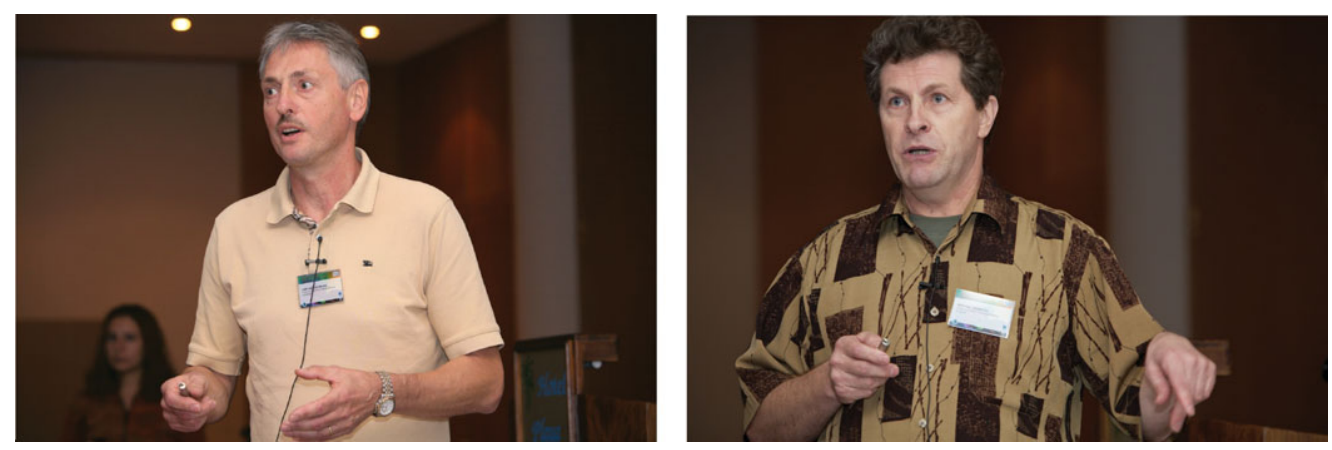

Uwe Motschmann (left), and Mikhail Demidov (right)
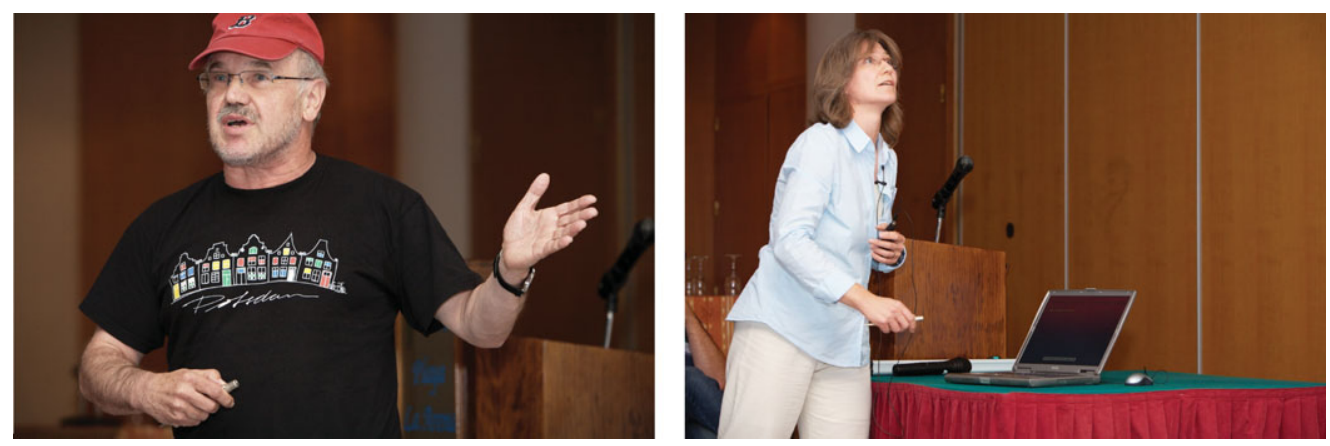

Hans Zinnecker (left), and Monika Petr-Gotzens (right)
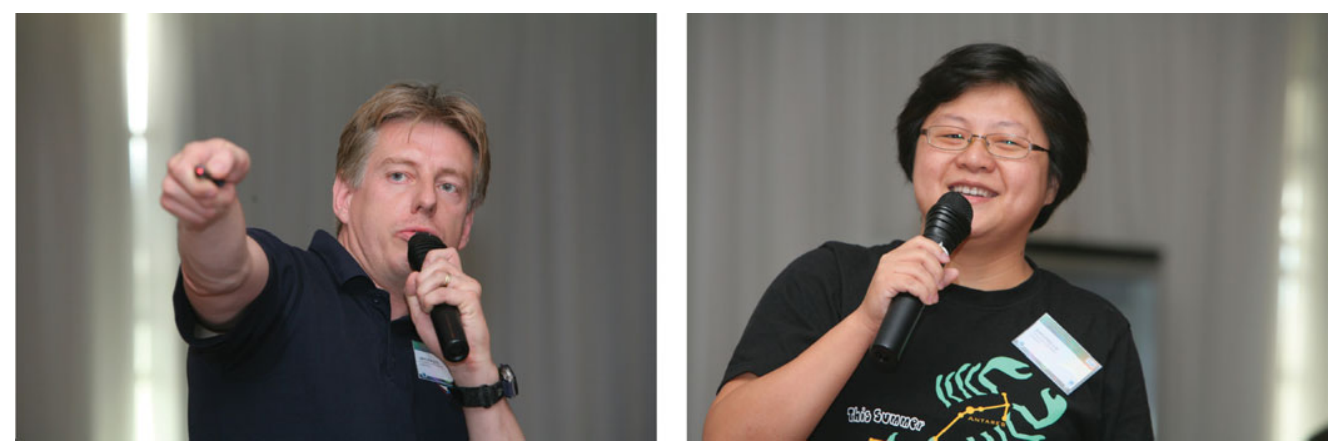

Jeroen Stil (left), and Shih-Ping Lai (right)
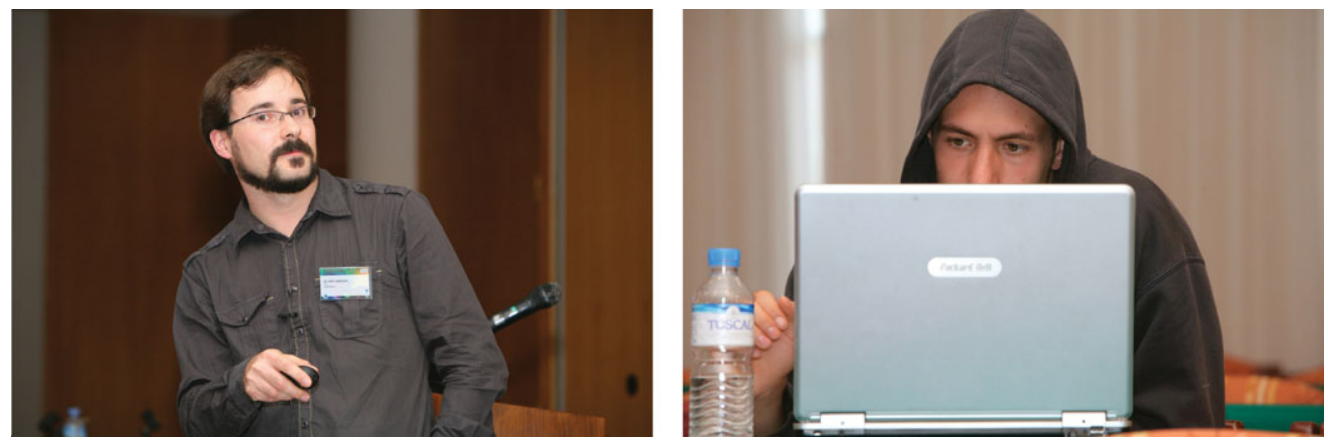

Oliver Gressel (left), and Darth Vader, private (right) 